\title{
Dosimetric comparison of organs at risk with three-dimensional conformal radiation, intensity-modulated radiation and volumetric- modulated arc therapy in cervical cancer: a cross sectional study
}

\author{
Febin Antony, Mathew Varghese K.*, Jomon Raphael C., Paul Gopu G., S. Sivakumar
}

Department of Radiation Oncology, Amala Institute of Medical Sciences, Thrissur, Kerala, India

Received: 07 February 2021

Accepted: 05 March 2021

\section{*Correspondence:}

Dr. Mathew Varghese K.,

E-mail: drmathew@hotmail.com

Copyright: ( ) the author(s), publisher and licensee Medip Academy. This is an open-access article distributed under the terms of the Creative Commons Attribution Non-Commercial License, which permits unrestricted non-commercial use, distribution, and reproduction in any medium, provided the original work is properly cited.

\begin{abstract}
Three-dimensional conformal radiation therapy (3DCRT), intensity-modulated radiation therapy (IMRT) and volumetric-modulated arc therapy (VMAT) are the three main radiotherapy treatment techniques for cervical cancer. Whether either technique significantly reduces the radiation exposure to organs at risk remains unclear. We dosimetrically compared the irradiated volumes of bone marrow, bladder and rectum in cervical cancer patients using 3DCRT, IMRT and VMAT techniques in those patients with FIGO stage IIIB cervical cancer, receiving chemo irradiation at our institute. A total of 10 patients were dosimetrically compared. Significant reduction in V10, V20, V30, V40, V50 Gy of bone marrow was observed with IMRT and VMAT when compared to 3DCRT. Similar results were seen with V20, V30, V40, V50 Gy of bladder, and V40, V50 Gy of rectum. While comparing IMRT and VMAT, statistically significant dose reduction was noted in V20 Gy of bone marrow and V20 and V30 Gy of bladder with VMAT. When compared with 3DCRT the use of IMRT and/or VMAT reduced the radiation exposure to bone marrow, bladder, and rectum volumes at various radiation dose levels. VMAT can further reduce the radiation exposure to bone marrow and bladder when compared with IMRT. Thus, we propose the use of VMAT in cervical cancer to reduce the OAR toxicities.
\end{abstract}

Keywords: Cervical cancer, VMAT, IMRT, 3DCRT

\section{INTRODUCTION}

Pelvic external beam radiotherapy (RT) along with concurrent chemotherapy followed by intracavitary brachytherapy is the standard approach for locally advanced cervical carcinoma. ${ }^{1}$ Previously, twodimensional radiotherapy (2DRT) treatment planning on plain film $\mathrm{x}$-rays were performed with anteroposterior/posteroanterior or 4-field techniques. Later, with CT treatment planning, three-dimensional conformal radiation therapy (3DCRT) helped in better delineation of target volumes and organs at risk (OARs) with a higher ability to protect normal structures while using standard beam configurations. ${ }^{2}$ Thereafter IMRT achieved optimal coverage of the target volumes with sparing of OARs by the modulation of multiple treatment fields or arcs by inverse planning. ${ }^{3}$ Previous dosimetric studies have demonstrated decreased volumes of the bladder, rectum, bowel, and bone marrow doses in treatment of cervical cancer using IMRT. ${ }^{3}$ Similarly, there are prospective randomized trials and meta-analysis demonstrating decreased acute gastrointestinal and urinary toxicities with IMRT compared to 3DCRT..$^{4-7}$ Volumetric modulated arc therapy (VMAT), a sophisticated form of IMRT that delivers greater conformity and reduces treatment time compared with IMRT and VMAT can shorten treatment delivery time by $54 \%$ compared with fixed-field IMRT in cervical cancer radiotherapy. ${ }^{8}$ This superiority is particularly important as it improves patient comfort and compliance to the treatment. Studies revealed 
similar target volume coverage with better OAR protection with VMAT which provided the rationale for the use of VMAT in cervical cancer. ${ }^{9,10}$ As there are data on various techniques, we tried to dosimetrically compare the irradiated volumes of bladder, bone marrow and rectum in cervical cancer patients using 3DCRT, IMRT, and VMAT techniques for this case series.

\section{CASE SERIES}

Patients receiving chemo irradiation for cervical cancer at Amala Institute of Medical Sciences from March 2020 to July 2020 were included in this pilot study. Eligibility criteria included age $<70$ years of age, performance status Eastern co-operative oncology group $<2$, and a FIGO stage IIIB Adeno carcinoma or squamous cell carcinoma. Those patients with pyometra or hydrometra, had undergone previous pelvic surgery or systemic chemotherapy were excluded. At our institution, these patients are offered external beam radiotherapy with IMRT technique, total prescribed dose of 50.4 Gy in 28 fractions (1.8 Gy per fraction) along with weekly concurrent cisplatin (40 $\mathrm{mg} / \mathrm{m}^{2}$ ) I.V., followed by HDR brachytherapy of $7 \mathrm{~Gy}$ in 3 fractions prescribed to point $\mathrm{A}$.

On the simulation day, after getting the informed consent, patients were asked to empty the bladder first and to drink $500 \mathrm{ml}$ of water. After 45 minutes a contrast enhanced computed tomography (CT) scan of $3 \mathrm{~mm}$ slice thickness with ray cast immobilisation in supine position was taken for planning. Within the treatment planning system (Eclipse version 11.0), GTV (gross tumor volume), CTV (clinical target volume), PTV (planning target volume), and organs at risk (OAR)-rectum, bladder, bone marrow, bowel bag and bilateral femoral heads were delineated as per consensus guidelines. The prescribed external beam radiotherapy dose was 50.4 Gy in 28 fractions. For each patient 3 different plans were generated with 3DCRT, IMR, and VMAT. Data from the dose-volume histogram (DVH) obtained from all the three different plans were dosimetrically compared. The plan comparisons were focused on V10, V20, V30, V40 and V50 Gy to bladder, rectum, and bone marrow.

Continuous data represented in mean and standard deviation. Significance level was assessed at 5\% level. Correlation between the two continuous variables was assessed by Pearson correlation coefficient. ANOVA and unpaired $\mathrm{t}$ tests were used to compare the dosimetric parameters among the various plans.

A total of 10 patients having stage IIIB cervical carcinoma were studied. Dosimetric parameters for bladder, bone marrow, and rectum were analysed with 3DCRT, IMRT, and VMAT for individual patient. A statistically significant reduction in V10, V20, V30, V40 and V50 Gy of bone marrow was observed with IMRT and VMAT when compared to 3DCRT (V10 Gy; p=0.001, V20 Gy; $\mathrm{p}=0.0001, \mathrm{~V} 30$ Gy; $\mathrm{p}=0.0001, \mathrm{~V} 40 \mathrm{~Gy} ; \mathrm{p}=0.0001$, V50 $\mathrm{Gy} ; \mathrm{p}=0.0001$ ). Similar reduction was seen in V20, V30, V40 and V50 Gy of bladder (V20 Gy; p=0.0001, V30 Gy; $\mathrm{p}=0.0001$, V40 Gy; p=0.0001, V50 Gy; p=0.0001). No dose reduction was noted for V10, V20, V30 Gy of rectum, whereas significant dose reduction was noted for $\mathrm{V} 40$ and V50 Gy (V10 Gy; p=0.94, V20 Gy; p=0.93, V30 Gy; $\mathrm{p}=0.630, \mathrm{~V} 40 \mathrm{~Gy} ; \mathrm{p}=0.0001, \mathrm{~V} 50 \mathrm{~Gy} ; \mathrm{p}=0.0001$ ) shown in the Table 1.

While comparing IMRT and VMAT, statistically significant dose reduction was noted in V20 Gy of bone marrow ( $\mathrm{p}=0.002), \mathrm{V} 20$ and V30 Gy of bladder (V20 Gy; $\mathrm{p}=0.004$, V30 Gy; $\mathrm{p}=0.001$ ) with use of VMAT. No significant dose reduction was noted for rectal dose shown in the Table 2.

Table 1: Irradiated volumes of bone marrow, bladder and rectum in cervical cancer patients using 3DCRT, IMRT and VMAT.

\begin{tabular}{|c|c|c|c|c|c|c|c|c|c|c|}
\hline \multirow{2}{*}{ Dose } & \multirow{2}{*}{ Treatment } & \multicolumn{3}{|c|}{ Bone marrow } & \multicolumn{3}{|c|}{ Bladder } & \multicolumn{3}{|c|}{ Rectum } \\
\hline & & Mean & S. D. & $P$ value & Mean & S D & $P$ value & Mean & S. D. & $P$ value \\
\hline \multirow{3}{*}{ V10 } & 3DCRT & 95.58 & 3.32 & \multirow{3}{*}{0.001} & 100 & 0.00 & \multirow{3}{*}{-} & 97.81 & 5.81 & \multirow{3}{*}{0.943} \\
\hline & IMRT & 91.61 & 1.91 & & 100 & 0.00 & & 96.86 & 7.78 & \\
\hline & VMAT & 90.93 & 1.96 & & 100 & 0.00 & & 96.85 & 7.74 & \\
\hline \multirow{3}{*}{ V20 } & 3DCRT & 92.20 & 3.93 & \multirow{3}{*}{0.0001} & 99.59 & 0.95 & \multirow{3}{*}{0.0001} & 97.37 & 6.68 & \multirow{3}{*}{0.934} \\
\hline & IMRT & 79.72 & 1.14 & & 79.96 & 9.82 & & 96.46 & 8.49 & \\
\hline & VMAT & 76.62 & 2.48 & & 94.43 & 6.76 & & 96.07 & 8.97 & \\
\hline \multirow{3}{*}{ V30 } & 3DCRT & 73.41 & 7.27 & \multirow{3}{*}{0.0001} & 99.59 & 0.91 & \multirow{3}{*}{0.0001} & 97.10 & 7.13 & \multirow{3}{*}{0.630} \\
\hline & IMRT & 60.29 & 2.54 & & 79.96 & 9.82 & & 95.21 & 8.80 & \\
\hline & VMAT & 59.66 & 2.80 & & 94.43 & 6.76 & & 93.40 & 9.51 & \\
\hline \multirow{3}{*}{ V40 } & 3DCRT & 52.94 & 5.67 & \multirow{3}{*}{0.0001} & 87.30 & 15.48 & \multirow{3}{*}{0.0001} & 94.18 & 7.96 & \multirow{3}{*}{0.0001} \\
\hline & IMRT & 37.34 & 3.55 & & 54.45 & 17.20 & & 72.98 & 10.51 & \\
\hline & VMAT & 36.55 & 3.97 & & 58.35 & 18.19 & & 73.73 & 8.32 & \\
\hline \multirow{3}{*}{ V50 } & 3DCRT & 31.66 & 7.50 & \multirow{3}{*}{0.0001} & 78.81 & 18.58 & \multirow{3}{*}{0.0001} & 81.17 & 9.43 & \multirow{3}{*}{0.0001} \\
\hline & IMRT & 15.19 & 3.32 & & 27.73 & 17.59 & & 29.90 & 9.72 & \\
\hline & VMAT & 14.91 & 3.61 & & 28.39 & 15.34 & & 34.28 & 12.45 & \\
\hline
\end{tabular}


Table 2: Irradiated volumes of bone marrow, bladder and rectum in cervical cancer patients using IMRT and VMAT.

\begin{tabular}{|c|c|c|c|c|c|c|c|c|c|c|}
\hline \multirow{2}{*}{ Dose } & \multirow{2}{*}{ Treatment } & \multicolumn{2}{|c|}{ Bone marrow } & \multicolumn{3}{|c|}{ Bladder } & \multirow[b]{2}{*}{$P$ value } & \multicolumn{2}{|c|}{ Rectum } & \multirow[b]{2}{*}{$P$ value } \\
\hline & & Mean & S. D. & $P$ value & Mean & S. D. & & Mean & S. D. & \\
\hline \multirow{2}{*}{ V10 } & IMRT & 91.61 & 1.91 & \multirow{2}{*}{0.445} & 100 & 0.00 & & 96.86 & 7.78 & \multirow{2}{*}{0.997} \\
\hline & VMAT & 90.93 & 1.96 & & 100 & 0.00 & & 96.85 & 7.74 & \\
\hline \multirow{2}{*}{ V20 } & IMRT & 79.72 & 1.148 & \multirow{2}{*}{0.002} & 97.73 & 2.10 & \multirow{2}{*}{0.004} & 96.46 & 8.49 & \multirow{2}{*}{0.921} \\
\hline & VMAT & 76.62 & 2.48 & & 99.94 & 0.17 & & 96.07 & 8.97 & \\
\hline \multirow{2}{*}{ V30 } & IMRT & 60.29 & 2.54 & \multirow{2}{*}{0.605} & 79.96 & 9.82 & \multirow{2}{*}{0.001} & 95.21 & 8.80 & \multirow{2}{*}{0.663} \\
\hline & VMAT & 59.66 & 2.80 & & 94.43 & 6.76 & & 93.40 & 9.51 & \\
\hline \multirow{2}{*}{ V40 } & IMRT & 37.34 & 3.55 & \multirow{2}{*}{0.644} & 54.45 & 17.20 & \multirow{2}{*}{0.628} & 72.98 & 10.51 & \multirow{2}{*}{0.861} \\
\hline & VMAT & 36.55 & 3.97 & & 58.35 & 18.19 & & 73.73 & 8.32 & \\
\hline \multirow{2}{*}{ V50 } & IMRT & 15.19 & 3.32 & \multirow{2}{*}{0.859} & 27.73 & 17.59 & \multirow{2}{*}{0.931} & 29.90 & 9.72 & \multirow{2}{*}{0.392} \\
\hline & VMAT & 14.91 & 3.61 & & 28.39 & 15.34 & & 34.28 & 12.45 & \\
\hline
\end{tabular}

\section{DISCUSSION}

The standard of carcinoma cervix stage $1 \mathrm{~B} 3$ to IVA is definitive chemoradiotherapy. ${ }^{1}$ The technique of delivering radiotherapy has improved from the box field to CT based highly conformal modalities. ${ }^{11}$ 3DCRT has a greater ability to protect OAR through more precise blocking while using standard beam configurations where as in IMRT and VMAT with modulation of multiple treatment fields or arcs optimal coverage of the target volume with sparing of OARs can be achieved by inverse planning. ${ }^{12}$

Few prospective randomized trials and one meta-analysis has demonstrated decreased acute gastrointestinal and urinary toxicities with IMRT compared to 3DCRT. ${ }^{4,5,13}$ Dosimetric studies has also shown reduced bone marrow toxicity with the use of IMRT. ${ }^{14}$ ASTRO clinical practice guidelines on radiation therapy for cervical cancer 2019 gives a strong recommendation for use of IMRT in postoperative case and a conditional recommendation for use of IMRT in definitive cases. ${ }^{18}$ Despite the significant benefits, there are a few shortcomings for IMRT. The use of multiple fixed-angle radiation beams can increase treatment delivery time which have effect on patient comfort, inter and intra-fraction motion. ${ }^{8}$ IMRT also uses a larger number of monitor units (MUs) when compared with 3DCRTthus increasing the amount of peripheral dose to other organs. ${ }^{8}$ VMAT, a newer radiation technique lets continuous delivery of radiation by varying the dose rate, the positions of the multi leaf collimator, and the gantry rotation speed simultaneously. Highly conformal dose distributions with improved target-volume coverage and normal tissues sparing can be achieved in VMAT. ${ }^{9}$ It also has the potential benefit of reduction in both treatment delivery time and MU usage when compared with IMRT. ${ }^{8}$ In our pilot study we dosimetrically compared the irradiated volumes of bladder, bone marrow and rectum in cervical cancer patients using 3DCRT, IMRT and VMAT.

The studies by Heron et al, Igdem et al and Roeske et al reported a significantly reduction in the irradiated volume of the rectum at doses of 30,40 and $45 \mathrm{~Gy}$, when comparing IMRT to 3D-CRT. ${ }^{16-18}$ A meta-analysis published in 2012 indicated that the pooled average percent volumes of irradiated rectum at doses of 30, 35, 40 and 45 Gy were significantly lower in IMRT when compared with 3DCRT. The reduction in rectal dose also manifested a dose response relationship with increasing radiation doses. Authors of the metanalysis concluded that IMRT can reduce the average percent of irradiated volumes to the rectum when higher radiation doses are used. ${ }^{19}$ In our study V40 Gy $(p=0.001)$ and V50 Gy $(\mathrm{p}=0.001)$ of rectum had a significant dose reduction using IMRT and VMAT than 3DCRT which was comparable to the above observations.

Meta-analysis published in 2012 could not find any statistically significant association with use of IMRT and reduction in the irradiated volumes of the bladder. ${ }^{19}$ Another metanalysis published in 2018 concluded that incidence of grade 3 or higher GU toxicity were lower in IMRT than 3DCRT. ${ }^{7}$ In our present study statistically significant reduction in V20 Gy $(\mathrm{p}=0.001)$, V30 Gy $(\mathrm{p}=0.001), \mathrm{V} 40 \mathrm{~Gy}(\mathrm{p}=0.001)$ and V50 Gy $(\mathrm{p}=0.001)$ was observed with IMRT and VMAT when compared to 3DCRT for bladder volume.

Brixey et al reported no obvious reduction in the irradiated volumes of bone marrow at doses of 10 and $30 \mathrm{~Gy}$ but had a statistically significant reduction for doses at 20, 40, and 45 Gy using IMRT. ${ }^{20}$ Few other studies have reported reduction in bone marrow volume at several high radiation levels delivered by IMRT. ${ }^{21,22}$ Meta-analysis published in 2012 concluded that IMRT reduced the average percent volumes of irradiated bone marrow at all radiation doses, but without a statistical significance ${ }^{[19]}$. In our study statistically significant reduction in V10 Gy $(\mathrm{p}=0.001)$, V20 Gy $(\mathrm{p}=0.001)$, V30 Gy (p=0.001), V40 Gy (p=0.001), and V50 Gy $(\mathrm{p}=0.001)$ was observed with IMRT and VMAT when compared to 3DCRT for bone marrow volume.

Metanalysis published in 2018 had dosimetrically compared the irradiated volumes of OAR with IMRT and 
VMAT. Authors concluded that for rectum V40 Gy was significantly decreased, but no significant differences were observed for bladder. ${ }^{23}$ In our study the use of VMAT had statistically significant dose reduction in V20 Gy of bone marrow ( $\mathrm{p}=0.002), \mathrm{V} 20$ and V30 Gy of bladder (V20 Gy; $\mathrm{p}=0.004$, V30 Gy; $\mathrm{p}=0.001)$.

With the available evidence and our study, we found that the use of IMRT and VMAT reduced the dose to bone marrow, bladder, and rectum when compared to 3DCRT. The use of VMAT can further reduce the dose to bladder and bone marrow. VMAT also has the advantage of completing the per day treatment at lesser time than IMRT. ${ }^{8}$ This reduction in treatment time can be utilised for proper image verification which can minimize the treatment uncertainties and reduce the risks of target miss which is considered as the biggest problem with IMRT. ${ }^{24}$

\section{CONCLUSION}

When compared with 3DCRT the use of IMRT and VMAT reduced the radiation exposure to bone marrow, bladder, and rectum volumes at various radiation dose levels. VMAT can further reduce the radiation exposure to bone marrow and bladder when compared with IMRT, thus we propose the use of VMAT in cervical cancer to reduce the OAR toxicities.

\section{ACKNOWLEDGEMENTS}

The authors acknowledge with gratitude the immense help given by Dr. P. C. Sudheeran, and Mrs. Jini M. P. during all stages of the work.

\section{Funding: No funding sources Conflict of interest: None declared Ethical approval: Not required}

\section{REFERENCES}

1. NCCN Clinical Practice Guidelines in Oncology. Available at: https://www.nccn.org/professionals/ physician_gls/default.aspx. Accessed on 20 April 2020.

2. Gerstner N, Wachter S, Knocke TH, Fellner C, Wambersie A, Pötter R. The benefit of Beam's eye view-based 3D treatment planning for cervical cancer. Radiother Oncol. 1999;51:71-8.

3. Du XL, Tao J, Sheng XG, Lu CH, Yu H, Wang C, et al. Intensity-modulated radiation therapy for advanced cervical cancer: a comparison of dosimetric and clinical outcomes with conventional radiotherapy. Gynecol Oncol. 2012;125:151-7.

4. Gandhi AK, Sharma DN, Rath GK, Julka PK, Subramani V, Sharma S, et al. Early clinical outcomes and toxicity of intensity modulated versus conventional pelvic radiation therapy for locally advanced cervix carcinoma: a prospective randomized study. Int J Radiat Oncol Biol Phys. 2013;87:542-8.
5. Naik A, Gurjar OP, Gupta KL, Singh K, Nag P, Bhandari V. Comparison of dosimetric parameters and acute toxicity of intensity-modulated and threedimensional radiotherapy in patients with cervix carcinoma: A randomized prospective study. Cancer Radiother. 2016;20:370-6.

6. Yu C, Zhu W, Ji Y, Guo J, Pan P, Han J, et al. A comparative study of intensity-modulated radiotherapy and standard radiation field with concurrent chemotherapy for local advanced cervical cancer. Eur J Gynaecol Oncol. 2015;36:278-82.

7. Lin Y, Chen K, Lu Z, Zhao L, Tao Y, Ouyang Y, et al. Intensity-modulated radiation therapy for definitive treatment of cervical cancer: a metaanalysis. Radiat Oncol. 2018;13:177.

8. Jia MX, Zhang X, Yin C, Feng G, Li N, Gao S, et al. Peripheral dose measurements in cervical cancer radiotherapy: a comparison of volumetric modulated arc therapy and step-and-shoot IMRT techniques. Radiat Oncol. 2014;9:61.

9. Cozzi L, Dinshaw KA, Shrivastava SK, Mahantshetty U, Engineer R, Deshpande DD et al. A treatment planning study comparing volumetric arc modulation with RapidArc and fixed field IMRT for cervix uteri radiotherapy. Radiother Oncol. 2008;89:180-91.

10. Knapp P, Eva B, Reseigh G, Gibbs A, Sim L, Daly T, et al. The role of volumetric modulated arc therapy (VMAT) in gynaecological radiation therapy: A dosimetric comparison of intensity modulated radiation therapy versus VMAT. J Med Radiat Sci. 2019;66:44-53.

11. Bucci MK, Bevan A, Roach M. $3^{\text {rd }}$ Advances in radiation therapy: conventional to $3 \mathrm{D}$, to IMRT, to 4D, and beyond. CA Cancer J Clin. 2005;55:117-34.

12. Guy JB, Falk AT, Auberdiac P, Cartier L, Vallard A, Ollier E et al. Dosimetric study of volumetric arc modulation with RapidArc and intensity-modulated radiotherapy in patients with cervical cancer and comparison with 3-dimensional conformal technique for definitive radiotherapy in patients with cervical cancer. Med Dosim. 2016;41:9-14.

13. Lin AJ, Kidd E, Dehdashti F, Siegel BA, Mutic S, Thaker PH et al. Intensity Modulated Radiation Therapy and Image-Guided Adapted Brachytherapy for Cervix Cancer. Int J Radiat Oncol Biol Phys. 2019;103:1088-97.

14. Mell LK, Sirák I, Wei L, Tarnawski R, Mahantshetty $\mathrm{U}$ et al. INTERTECC Study Group. Bone Marrowsparing Intensity Modulated Radiation Therapy with Concurrent Cisplatin For Stage IB-IVA Cervical Cancer: An International Multicenter Phase II Clinical Trial (INTERTECC-2). Int J Radiat Oncol Biol Phys. 2017;97:536-45.

15. Chino J, Annunziata CM, Beriwal S, Bradfield L, Erickson BA, Fields EC et al. Radiation Therapy for Cervical Cancer: Executive Summary of an ASTRO Clinical Practice Guideline. Pract Radiat Oncol. 2020;10:220-34.

16. Heron DE, Gerszten K, Selvaraj RN, King GC, Sonnik D, Gallion $\mathrm{H}$, et al. Conventional 3D 
conformal versus intensity-modulated radiotherapy for the adjuvant treatment of gynecologic malignancies: a comparative dosimetric study of dose-volume histograms. Gynecol Oncol. 2003;91:39-45.

17. Iğdem S, Ercan T, Alço G, Zengin F, Ozgüleş R, Geceer G, et al. Dosimetric comparison of intensity modulated pelvic radiotherapy with $3 \mathrm{D}$ conformal radiotherapy in patients with gynecologic malignancies. Eur J Gynaecol Oncol. 2009;30:54751.

18. Roeske JC, Lujan A, Rotmensch J, Waggoner SE, Yamada D, Mundt AJ. Intensity-modulated whole pelvic radiation therapy in patients with gynecologic malignancies. Int $\mathbf{J}$ Radiat Oncol Biol Phys. 2000;48:1613-21.

19. Yang B, Zhu L, Cheng H, Li Q, Zhang Y, Zhao Y. Dosimetric comparison of intensity modulated radiotherapy and three-dimensional conformal radiotherapy in patients with gynecologic malignancies: a systematic review and meta-analysis. Radiat Oncol. 2012;7:197.

20. Brixey CJ, Roeske JC, Lujan AE, Yamada SD, Rotmensch J, Mundt AJ. Impact of intensitymodulated radiotherapy on acute hematologic toxicity in women with gynecologic malignancies. Int J Radiat Oncol Biol Phys. 2002;54:1388-96.
21. Ahmed RS, Kim RY, Duan J, Meleth S, De Los Santos JF, Fiveash JB. IMRT dose escalation for positive para-aortic lymph nodes in patients with locally advanced cervical cancer while reducing dose to bone marrow and other organs at risk. Int J Radiat Oncol Biol Phys. 2004;60:505-12.

22. Lujan AE, Mundt AJ, Yamada SD, Rotmensch J, Roeske JC. Intensity-modulated radiotherapy as a means of reducing dose to bone marrow in gynecologic patients receiving whole pelvic radiotherapy. Int $\mathrm{J}$ Radiat Oncol Biol Phys. 2003;57:516-21.

23. Bai W, Kou C, Yu W, Li Y, Hua W, Yu L, et al. Dosimetric comparison of volumetric-modulated arc therapy and intensity-modulated radiation therapy in patients with cervical cancer: a meta-analysis. Onco Targets Ther. 2018;11:7179-86.

24. Ríos I, Vásquez I, Cuervo E, Garzón Ó, Burbano J. Problems and solutions in IGRT for cervical cancer. Rep Pract Oncol Radiother. 2018;23:517-27.

Cite this article as: Antony F, Mathew VK, Jomon RC, Paul GG, Sivakumar S. Dosimetric comparison of organs at risk with three-dimensional conformal radiation, intensity-modulated radiation and volumetric-modulated arc therapy in cervical cancer: a case series. Int J Adv Med 2021;8:586-90. 